\title{
Unruptured Right Sinus of Valsalva Aneurysm Dissecting into Interventricular Septum Causing Complete Heart Block: Can Early Surgical Correction Revert Rhythm Disturbances?
}

\author{
Prerit Agarwal', Ankit Jain'1, Pawan Singh', Harpreet Singh', Muhammad Abid Geelani', \\ Vimal Mehta ${ }^{2}$ \\ ${ }^{1}$ Department of Cardiothoracic and Vascular Surgery, Govind Ballabh Pant Institute of Post Graduate Medical Education and \\ Research, Delhi University, New Delhi, India \\ ${ }^{2}$ Department of Cardiology, Govind Ballabh Pant Institute of Post Graduate Medical Education and Research, Delhi University, \\ New Delhi, India \\ Email: agarwal.cvts@gmail.com
}

How to cite this paper: Agarwal, P., Jain, A., Singh, P., Singh, H., Geelani, M.A. and Mehta, V. (2018) Unruptured Right Sinus of Valsalva Aneurysm Dissecting into Interventricular Septum Causing Complete Heart Block: Can Early Surgical Correction Revert Rhythm Disturbances? World Journal of Cardiovascular Diseases, 8, 353-359. https://doi.org/10.4236/wjcd.2018.87034

Received: May 28, 2018

Accepted: July 22, 2018

Published: July 25, 2018

Copyright $\odot 2018$ by author and Scientific Research Publishing Inc. This work is licensed under the Creative Commons Attribution International License (CC BY 4.0).

http://creativecommons.org/licenses/by/4.0/

\section{(c) (i) Open Access}

\begin{abstract}
A sinus of Valsalva aneurysm (SOVA) is abnormal dilatation of the either aortic sinuses, area of the aortic root between the aortic valve annulus and the sinotubular junction. Their clinical presentation may range from being asymptomatic as an incidental finding on cardiac imaging to symptomatic presentations related to the compression of adjoining structures or intracardiac shunting caused by rupture of the SOVA mostly into the right side of the heart. The compression leads to findings of tricuspid valve regurgitation, right ventricular outflow tract (RVOT) obstruction and rarely complete heart block (CHB). Dissection or erosion into interventricular septum is one of the rarest complications of SOVA. The symptomatic presentation is almost always a surgical emergency. Here we present a case report of a patient with unruptured sinus of valsalva originating from right sinus dissecting into interventricular septum causing complete heart bock. In this case after surgical correction the complete heart block reverted to sinus rhythm.
\end{abstract}

\section{Keywords}

Sinus of Valsalva Aneurysm (SOVA), Complete Heart Block (CHB)

\section{Introduction}

A sinus of Valsalva aneurysm (SOVA) is abnormal dilatation of the either aortic 
sinuses, area of the aortic root between the aortic valve annulus and the sinotubular junction. In a normal heart, out of the three sinuses, two sinuses contain origin of the coronary artery which are the left and right sinus, whereas the posterior sinus is a noncoronary sinus. Sinuses function to permit aortic valve opening during systole without the occlusion of coronary artery ostia. Sinus diameter varies by gender. The anatomic positioning of each sinus within the heart is a major determinant of clinical outcome in the case of SOVA formation and/or rupture. The right sinus lies adjacent to the interventricular septum and the right ventricular parietal bands. The left sinus is proximal to the anterior left ventricular free wall as well as the anterior mitral leaflet. The noncoronary (posterior) sinus rests above the interventricular septum, a portion of the anterior mitral leaflet, and forms a complex with the transverse sinus. These aneurysms gradually progress and remain silent. Their clinical presentation may range from being asymptomatic as an incidental finding on cardiac imaging to symptomatic presentations related to the compression of adjoining structures or intracardiac shunting caused by rupture of the SOVA mostly into the right side of the heart [1]. The compression leads to findings of tricuspid valve regurgitation, right ventricular outflow tract (RVOT) obstruction and rarely complete heart block (CHB). Dissection or erosion into interventricular septum is one of the rarest complications of SOVA [1] [2]. The symptomatic presentation is almost always a surgical emergency.

CHB being a rare complication of unruptured sinus of valsalva, associated medical literature is scarce; hence more such cases need to be brought to notice.

\section{Case History}

We present a case of 52 year old gentleman, who came to our emergency with complaints of frequent episodes of dizziness for the last 2.5 months. On evaluation he was hemodynamically stable with an unremarkable physical examination except for bradycardia. His 12 lead electrocardiogram revealed a complete heart block with a ventricular escape rhythm of 32 beats/min for which transvenous pacing via femoral vein was instituted (Figure 1). A review of previous medical records revealed the patient to be a known case of coronary artery disease with a history of percutaneous transluminal coronary angioplasty to Left anterior descending coronary artery in 2015. Trans-thoracic echocardiography showed presence of an unruptured sinus of valsalva aneurysm arising from the right coronary sinus invading into the interventricular septum with mild aortic regurgitation and normal left ventricular function. Cardiac computed tomography confirmed the diagnosis of unruptured right sinus of valsalva aneurysm with dimensions of $5 \times 2.7 \times 5.9 \mathrm{~cm}$. dissecting into the interventricular septum (Figure 2 and Figure 3). Coronary angiogram was also done with showed insignificant stent stenosis of $50 \%$ with rest of coronary arteries devoid of any significant stenosis. The patient was taken up for surgical repair via median sternotomy with standard bicaval canulation and cardioplegic arrest. After aortotomy, the right sinus of valsava aneurysm was opened. The aneurysm was dissecting into the in- 
terventricular septum. The aneurysmal cavity was filled with mural clots and calcified deposits (Figure 4). The aneurysm was seen distorting the aortic annulus leading to poor coaptation of the aortic valve leaflets. For repair, direct closure of the aneurysm cavity was done and aortic valve was replaced with size 23 mm medtronic ATS mechanical aortic valve (Figure 5 and Figure 6). Patient was weaned of cardiopulmonary bypass normally. Post bypass patient required temporary pacing with epicardial pacing leads. Patient was weaned off ventilator support on day 1 and reverted to sinus rhythm on the same day with a heart rate of 92 beats/min (Figure 7). Patient was finally discharged with sable vitals and uneventful hospital stay. Patient is currently in our bi-annual follow up and symptom free with stable cardiac rhythm.

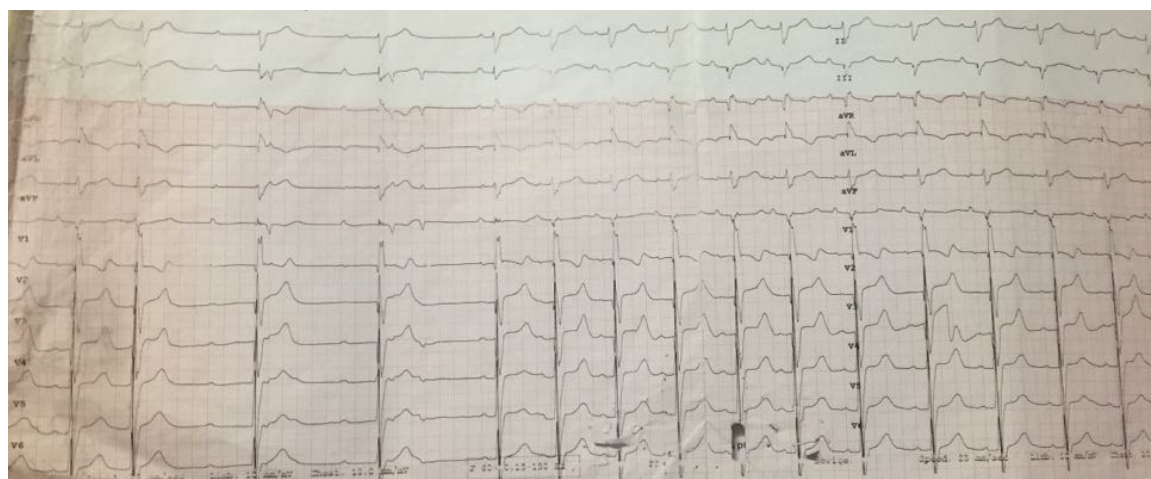

Figure 1. Electrocardiogram of the patient with and without transvenous pacing.

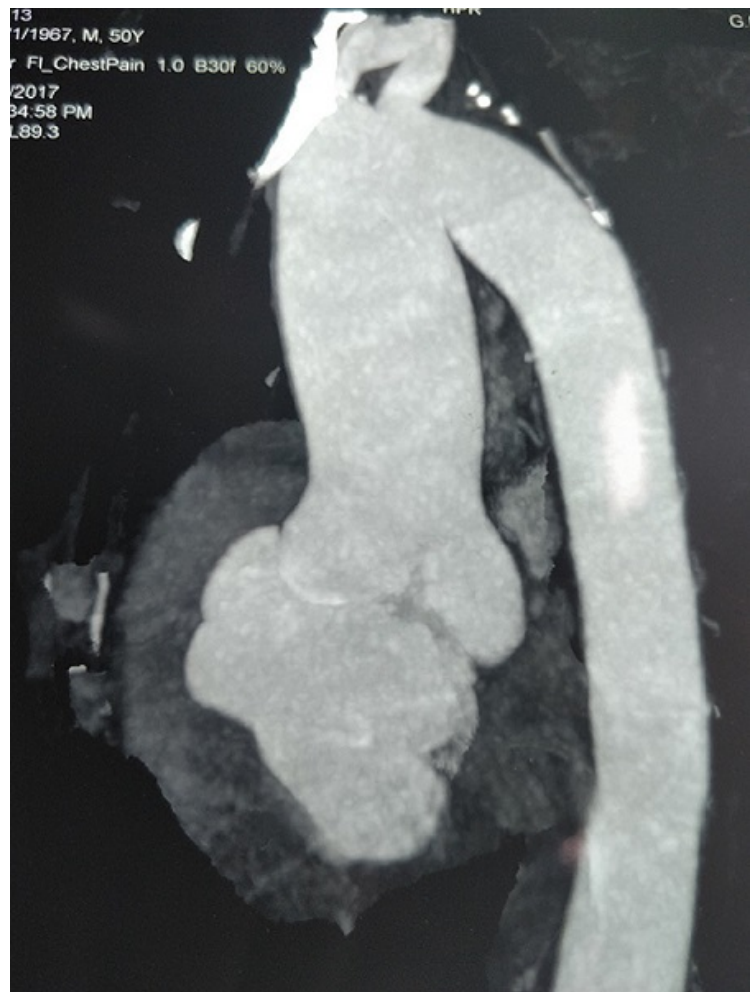

Figure 2. 2D CT figure of the unruptured aneurysm arising from right sinus of valsalva dissecting into IVS. 


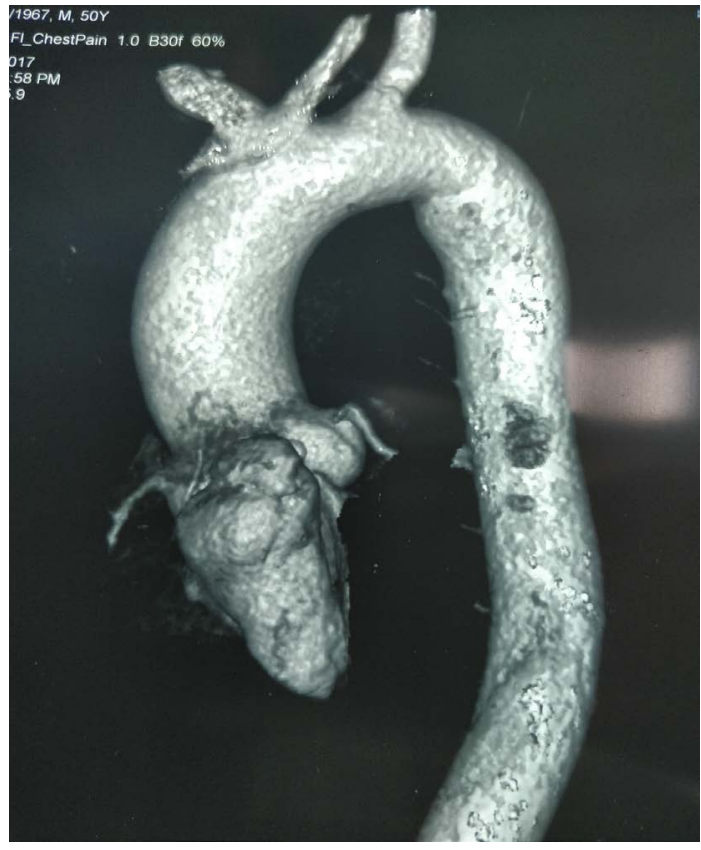

Figure 3. 3D CT figure unruptured aneurysm of sinus of valsalva.

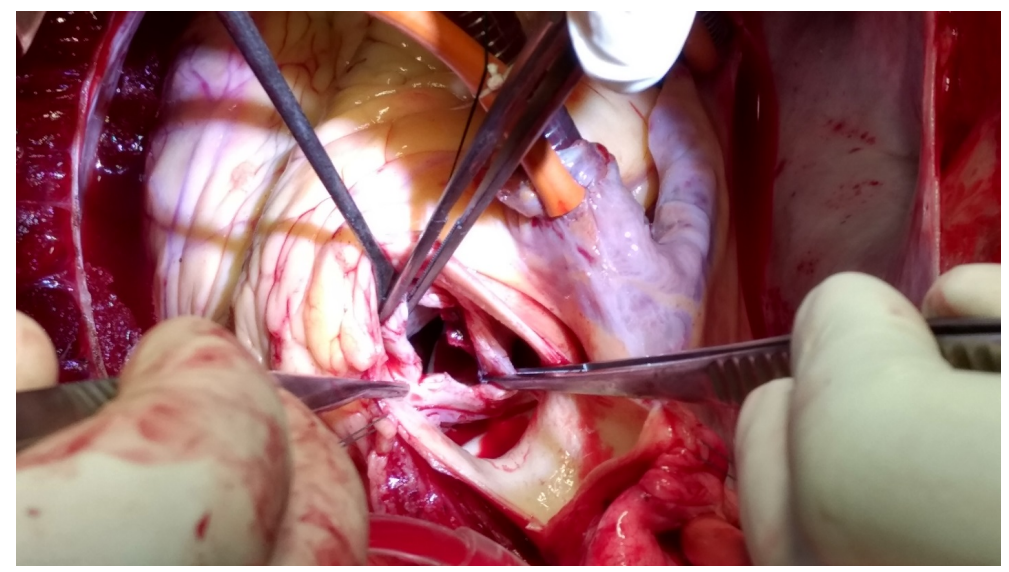

Figure 4. Intra operative view of the aneurysm.

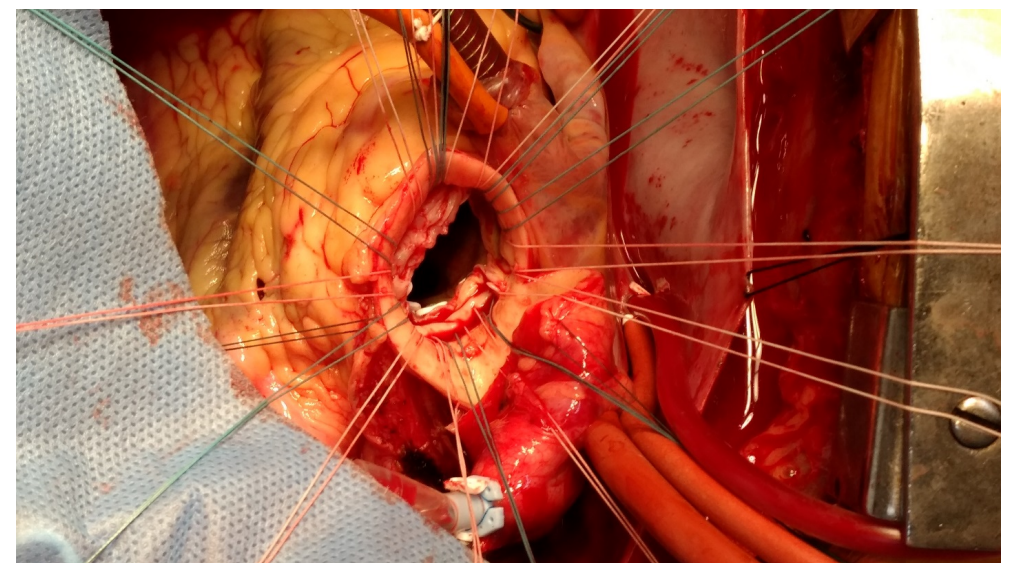

Figure 5. Intra operative view after primary repair of aneurysm. 


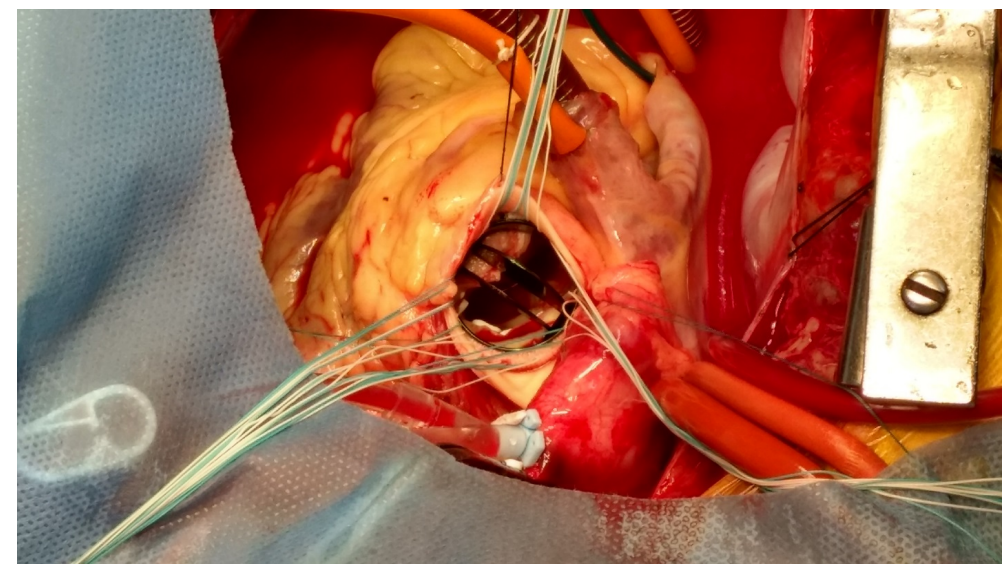

Figure 6. Intra operative view after mechanical aortic valve implantation.

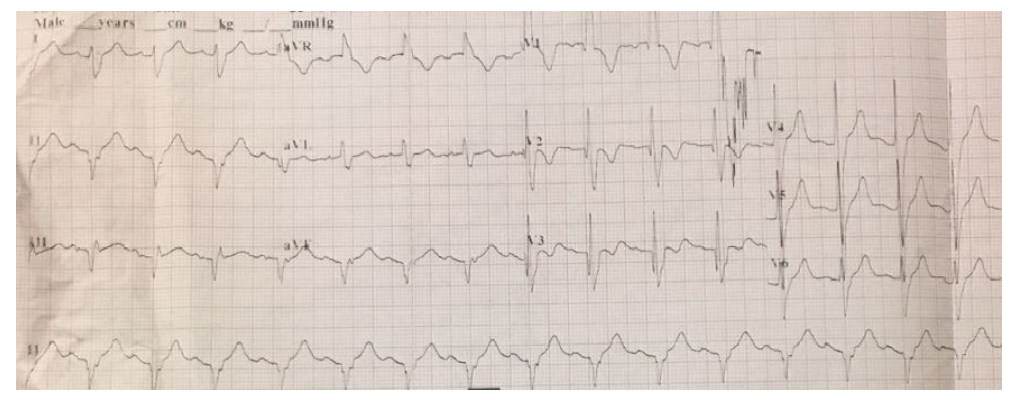

Figure 7. Post-operative day 3 electrocardiogram showing sinus rhythm.

\section{Discussion}

Sinus of valsalva aneurysm is a result of weakness of the elastic layer of aortic sinuses. It can be either a congenital or acquired deformity. The congenital variant is generally associated with connective tissue disorders like Marfan's syndrome and Ehler-Danos syndrome [3] [4]. The various causes in the acquired group may be infective (Tuberculosis, bacterial endocarditis, syphilis, and Behçet syndrome), trauma and degenerative pathology. The incidence of isolated SOVA in itself, is very low, reported at around $0.09 \%$ of the general population. The associated cardiac defects include bicuspid aortic valve (9\%), ventricular septal defect (34\%), and aortic regurgitation (44\%) [5]. SVAs are most frequently located in the right coronary sinus (67\% to $77 \%)$ with $15 \%$ originating in the non-coronary sinus and rare reports of isolated left SOVAs [6]. It has been reported that SOVA has a male preponderance of (4:1) [6]. The average age of presentation is 30 years and age range is $11-64$ years. The various clinical presentation of unruptured sinus of valsalva aneurysm includes compression of neighboring structures leading to RVOT obstruction, tricuspid valve regurgitation, aortic valve regurgitation, acute coronary syndrome and complete heart block by dissection into the interventricular septum [1]. While the ruptured aneurysm usually presents with sign and symptoms of acute heart failure due to large left to right shunt, the plausible explanation for complete heart block is the compression of the bundle of his and its branches by the dissecting aneurysm in 
the interventricular septum by mass effect. The diagnosis is usually incidental in unruptured sinus of valsalva aneurysms with echocardiogram being the first imaging modality. Cardiac catheterization and cardiac computed tomography are additional investigation needed for further evaluation and planning for the intervention needed. Ruptured sinus of valsalva aneurysm can be closed by surgery or device closure, provided margins are adequate. The symptomatic unruptured aneurysm always needs surgery. It is still rare to come across unruptured giant aneurysm with complete heart block, and these case reports are essential so as to formulate an effective protocol for the treatment strategy.

Gavali et al. reported a case with protrusion of aneurysm arising from the right coronary sinus (RCC) into the left ventricular outflow tract (LVOT) as well into the RVOT causing significant RVOT obstruction along with extension into the interventricular septum producing complete heart block. Their patient underwent uneventful surgery followed by permanent pacemaker insertion 2 weeks later [7].

Rajashekar et al. reported a case of SOVA arising from the left as well as right sinus causing transient complete heart block [8].

Barik et al. in his case report concluded that early surgery saves life in case of unruptured sinus of valsalva aneurysm and associated CHB may improve on follow up [9].

Gupta et al. reported a case of sinus of valsalva aneurysm arising from right as well as left sinus associated with CHB. After surgery the patient required a permanent pace maker implantation for improving CHB [10].

Our case is exceptional in the sense as the mass effect of a large unruptured sinus of valsalva included complete heart block with aortic valve regurgitation and with surgical repair the complete heart block reverted to sinus rhythm. To interpret that the noted complication, complete heart block can be reverted by operating as soon as possible on an unruptured sinus of valsalva aneurysm that has dissected into the interventricular septum is still debatable and more such case reports are required to reach a consensus.

\section{Funding}

This study has not been funded by any party whatsoever.

\section{Conflict of Interest}

All authors have no conflict of interest.

\section{Ethical Approval}

This article does not contain any studies with animals performed by any of the authors.

All procedures performed in study involving human participants were in accordance with the ethical standards of the institutional and/or national research committee and with the 1964 helsinki declaration and its later amendments or 
comparable ethical standards.

\section{Informed Consent}

Informed consent was obtained from all individual participants included in the study. Additional informed consent was obtained from all individual participants for whom identifying information is included in this article.

\section{References}

[1] Ring, W.S. (2000) Congenital Heart Surgery Nomenclature and Database Project: Aortic Aneurysm, Sinus of Valsalva Aneurysm, and Aortic Dissection. The Annals of Thoracic Surgery, 69, S147-S163. https://doi.org/10.1016/S0003-4975(99)01242-4

[2] Feldman, D.N. and Roman, M.J. (2006) Aneurysms of the Sinuses of Valsalva. Cardiology, 106, 73-81. https://doi.org/10.1159/000092635

[3] Ott, D.A. (2006) Aneurysm of the Sinus of Valsalva. Seminars in Thoracic and Cardiovascular Surgery. Pediatric Cardiac Surgery Annual, 9, 165-176. https://doi.org/10.1053/j.pcsu.2006.02.014

[4] Edwards, J.E. and Burchell, H.B. (1956) Specimen Exhibiting the Essential Lesion in Aneurysm of the Aortic Sinus. Proceedings of the Staff Meeting Mayo Clinic, 31, 407-412.

[5] Moustafa, S., Mookadam, F., Cooper, L., et al. (2007) Sinus of Valsalva Aneurysms-47 Years of a Single Center Experience and Systematic Overview of Published Reports. American Journal of Cardiology, 99, 1159-1164. https://doi.org/10.1016/j.amjcard.2006.11.047

[6] Sakakibara, S. and Konno, S. (1962) Congenital Aneurysms of Sinus of Valsalva. A Clinical Study. American Heart Journal, 63, 708-719. https://doi.org/10.1016/0002-8703(62)90018-2

[7] Gavali, S.A., Phadke, M.S., Kerkar, P.G., Nabar, A.A., Nyayadhish, P.Y. and Lanjewar, C.P. (2013) Unruptured Aneurysm of the Sinus of Valsalva Presenting with Right Ventricular Outflow Tract Obstruction, Complete Heart Block, and Protrusion into Left Ventricular Outflow Tract: A Rare Combination. Journal of the American College of Cardiology, 61, e169. https://doi.org/10.1016/j.jacc.2012.10.063

[8] Rajashekar, D., Subramanyam, G., Panchamukheswar, R., Praveen, M. and Guruprasad, S. (2005) Unruptured Sinus of Valsalva Aneurysms Manifesting as Complete Heart Block. Asian Cardiovascular and Thoracic Annals, 13, 283-286. https://doi.org/10.1177/021849230501300321

[9] Barik, R., et al. (2016) Giant Unruptured Sinus of Valsalva Aneurysm with Complete Heart Block. Journal of Cardiology Cases, 13, 17-20. https://doi.org/10.1016/j.jccase.2015.09.002

[10] Gupta, U., et al. (2016) Unruptured Aneurysm of Right Sinus of Valsalva Eroding Interventricular Septum Manifesting as Permanent Complete Heart Block. European Journal of Pharmaceutical and Medical Research, 3, 278-281. 